\title{
ENGELHARDT, Dietrich von, HARTMANN, Fritz, Klassiker der Medizin
}

Isabelle von Bueltzingsloewen

\section{OpenEdition}

Journals

Édition électronique

URL : http://journals.openedition.org/ifha/2060

DOI : $10.4000 /$ ifha. 2060

ISSN : 2198-8943

Éditeur

IFRA - Institut franco-allemand (sciences historiques et sociales)

Référence électronique

Isabelle von Bueltzingsloewen, « ENGELHARDT, Dietrich von, HARTMANN, Fritz, Klassiker der Medizin », Revue de I'IFHA [En ligne], Date de recension, mis en ligne le 01 janvier 1993, consulté le 22 septembre 2020. URL : http://journals.openedition.org/ifha/2060 ; DOI : https://doi.org/10.4000/ifha.2060

Ce document a été généré automatiquement le 22 septembre 2020

(CIFHA 


\title{
ENGELHARDT, Dietrich von, HARTMANN, Fritz, Klassiker der Medizin
}

\author{
Isabelle von Bueltzingsloewen
}

Cet ouvrage en deux volumes qui rassemble - les contributions de plus de trente auteurs, pour la plupart médecins historiens de la médecine, n'est pas sans susciter nombre de questions; il souligne en effet une fois de plus l'opposition qui existe - en Allemagne comme ailleurs - entre une histoire traditionnelle de la médecine qui est surtout histoire des sciences médicales et une histoire sociale de la médecine davantage préoccupée de restituer la réalité socio-politique, socio-économique et culturelle d'une époque et qui se veut également histoire de la santé, histoire de la maladie et histoire du corps et de ses représentations.

Il ne s'agit pas là de rejeter ou de critiquer la démarche biographique des deux éditeurs; dans ce domaine les instruments de travail sont déjà anciens et il eût été fort utile de les renouveler en tenant compte des acquis récents de la recherche concernant tel ou tel médecin ou tel ou tel courant médical. Mais telle n'est pas la démarche adoptée dans cet ouvrage. Son titre même pose problème en restreignant, dans une perspective très positiviste, l'entreprise aux seuls « classiques » de la médecine c'est-à-dire aux seuls médecins dont on estime qu'ils ont marqué la science médicale dans le sens du progrès. Le classement non pas alphabétique mais chronologique des notices, qui nous conduit du Ve siècle avant J.C à la fin des années 1950, de Hippocrate à Christoph Wilhelm Hufeland pour le tome 1 et de Philippe Pinel à Viktor von Weizsäcker pour le tome 2, confirme ce parti pris d'une histoire de la médecine conçue comme une succession de grandes figures. S'il est vrai qu'à l'intérieur de chacun des articles les auteurs se sont clairement donné pour objectif de replacer leur "personnage " dans le contexte politique et culturel de son temps - et il n'est nullement question ici de mettre en cause la qualité intrinsèque de chaque notice prise individuellement -, il ne s'agit pas pour autant d'une micro-histoire sociale de la médecine comme se plaisent à le dire F. v. 
Engelhardt et F. Hartmann dans une introduction longue d'à peine deux pages et demie.

Par ailleurs on ne peut que s'étonner de l'arbitraire du processus qui a conduit les auteurs à « sélectionner » tel ou tel médecin plutôt que tel autre: quels sont les critères qui donnent droit au label de " classique de la médecine »? Certes il nous est dit que l'entreprise est centrée sur l'espace allemand. Mais comment expliquer alors la présence entre Johann Peter Frank et Christoph Wilhelm Hufeland de Edward Jenner et de Marie-François-Xavier Bichat et celle de Philippe Pinel et de John Brown entre Hufeland et Samuel Hahnemann? Par ailleurs Bichat, Pinel et Laennec auraient-il davantage "mérité » de la médecine que Broussais ou Corvisard qui ne figurent pas dans l'ouvrage? Comment expliquer l'absence de Louis Pasteur à côté de Robert Koch?

$\mathrm{Au}$ total, on ne pourra que regretter le caractère peu novateur d'une entreprise qui au contraire accentue les traits d'une histoire traditionnelle de la médecine que la plupart des médecins se défendent pourtant de faire.

Isabelle von BUELTZINGSLOEWEN

INDEX

Thèmes : Ideengeschichte 